\title{
Topology Error Identification for the NEPTUNE Power System
}

\author{
Kevin Schneider, Student Member, IEEE, Chen-Ching Liu, Fellow, IEEE, and Bruce Howe, Member, IEEE
}

\begin{abstract}
The goal of the North Eastern Pacific Time-Series Undersea Networked Experiment (NEPTUNE) is to provide the infrastructure necessary for scientific exploration and investigation on the floor of the Pacific Ocean in an area encompassing the Juan de Fuca Tectonic Plate. In order to achieve this goal, the power delivery capabilities of the terrestrial distribution system will be extended into the Pacific Ocean. The power system associated with the proposed observatory is unlike conventional terrestrial power systems due to the unique under ocean operating conditions. The operating requirements of the system dictate hardware and software applications that are not found in terrestrial power systems. This paper describes a method for topology error identification in the presence of various forms of measurement errors for NEPTUNE, a highly interconnected DC power system. Hardware and reliability requirements have led to a power system configuration that includes a large number of unmeasured sections. Using previously developed state estimation algorithms as a starting point, a methodology for system topology identification is proposed in this paper.
\end{abstract}

Index Terms-DC power systems, state estimation, topology, underwater equipment, underwater technology.

\section{INTRODUCTION}

$\mathbf{S}$ UPPLYING power to under ocean electrical loads is currently at a transition point requiring a new operational paradigm [1]. Historically, powering under ocean experimentation has been a tradeoff between the power level and duration of the experiment. High power applications greater than a few watts generally require a ship-bound tether to supply the power. Due to the infeasibility of year round ship deployment, high power applications have been severely limited in duration. The NEPTUNE power system will contribute to the change of the operational paradigm of under ocean exploration and experimentation by providing science users with a continuous and relatively abundant supply of electrical power.

The NEPTUNE power system is a potential gateway to a new generation of power systems that are unlike any currently in operation. Effectively extending the terrestrial power system from the shoreline into an under ocean environment requires reexamining issues that have been studied extensively for terrestrial power systems.

Manuscript received February 2, 2004; revised December 24, 2004. This work was supported by the U.S. National Science Foundation: "Development of a power system for cabled ocean Observatories" OCE-0116750. Paper no. TPWRS-00047-2004.

K. Schneider and C.-C. Liu are with the Department of Electrical Engineering, University of Washington, Seattle, WA 98195-2500 USA (e-mail: ftla69@u.washington.edu; e-mail: liu@engr.washington.edu).

B. Howe is with the Applied Physics Laboratory, University of Washington, Seattle, WA 98195-2500 USA (e-mail: howe@apl.washington.edu).

Digital Object Identifier 10.1109/TPWRS.2005.851972
One of the significant operational challenges for the NEPTUNE power system will be to determine the status of power system breakers. Without knowledge of the correct breaker status control actions could cause damage to the system. To this end, state estimation techniques were developed that allow for the estimation of the un-metered backbone of the system [7].

The state estimation algorithms of [7] use redundant measurements, where available in the system, to estimate the voltages and currents in the sections of the system that are not directly measured. In a second stage of estimation, the voltages and currents that are directly measured are estimated. The difference between the direct measurement values and the estimated values are used to calculate a mean absolute residual. Post state estimation processing of the residual serves as the basis for determination of the breaker status. The proposed method will be able to identify single as well as multiple topological errors in the presence of Gaussian and gross measurement errors.

This paper is organized as follows. Section II presents an overview of the NEPTUNE power system. Included in this section is general information about the system and the arrangement of the physical components. Section III is an overview of the state estimation techniques that were developed and previously presented in [7]. The review of the state estimation technique is intended as a framework on which Section IV is built upon. Section IV presents the basis of the topology error identification method as well as the basic formulas. Section V gives a detailed overview of the process for determining the correct system topology. Section VI presents examples of the topology error identification method applied to the full NEPTUNE system.

\section{BACKGROUND}

The NEPTUNE system represents an example of a new form of ocean exploration, cabled observatories. Previous cabled observatories include numerous Japanese observatories used for disaster mitigation as well as various scientific based observatories in the United States [2].

Power systems for both terrestrial and under ocean applications such as cabled observatories have been deployed on large scales, but neither application addresses all requirements of NEPTUNE. Terrestrial power systems are based on interconnected alternating current (AC) networks with parallel loads, while underwater telecommunication systems are direct current (DC) point-to-point systems with series loads. The proposed NEPTUNE power system combines elements from each of these designs to create a system that differs significantly from either of the individual designs [3]-[6]. 


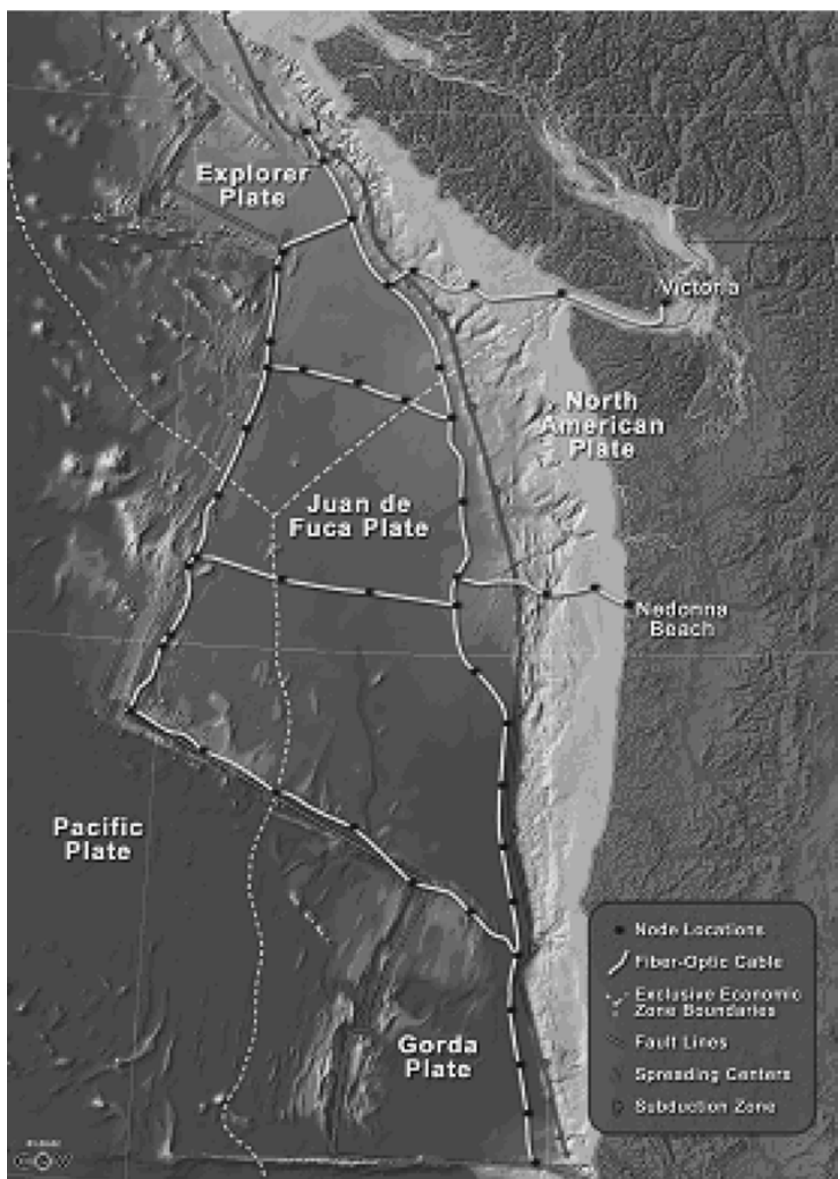

Fig. 1. NEPTUNE system.

In order to maximize the deliverable power, the back bone system will operate at $10 \mathrm{kV}$ with respect to the ocean. While the nominal $10-\mathrm{kV}$ voltage is well below standard terrestrial transmission and some distribution system voltages, it is the maximum rated voltage for standard under ocean telecommunications cables that will allow for the required 30-year life span [1].

Power is supplied to the system at the nominal $10-\mathrm{kV}$ voltage from two shore stations, one in the state of Oregon and the other in the province of British Columbia. The two shore stations will form the interface between the terrestrial power distribution system and the NEPTUNE power system. At the two shore stations the nominal voltage will be maintained through the use of AC-DC converters in conjunction with uninterruptible power supplies.

Each of the forty-six node locations in Fig. 1 will contain a node Branching Unit (BU), which branches the main cable via a spur (see Fig. 2). The spur may be up to several kilometers long, depending on the water depth at a particular location, and will supply power and communications to the science nodes.

Loads at the science node are served by pulse width modulated DC-to-DC converters delivering a stable 400 and $48 \mathrm{~V}$ from the incoming BU. Due to the operational characteristics of the converters at the science nodes, the loads at the science node have constant power characteristics. As a result, changes in system voltage do not significantly impact the power consumed

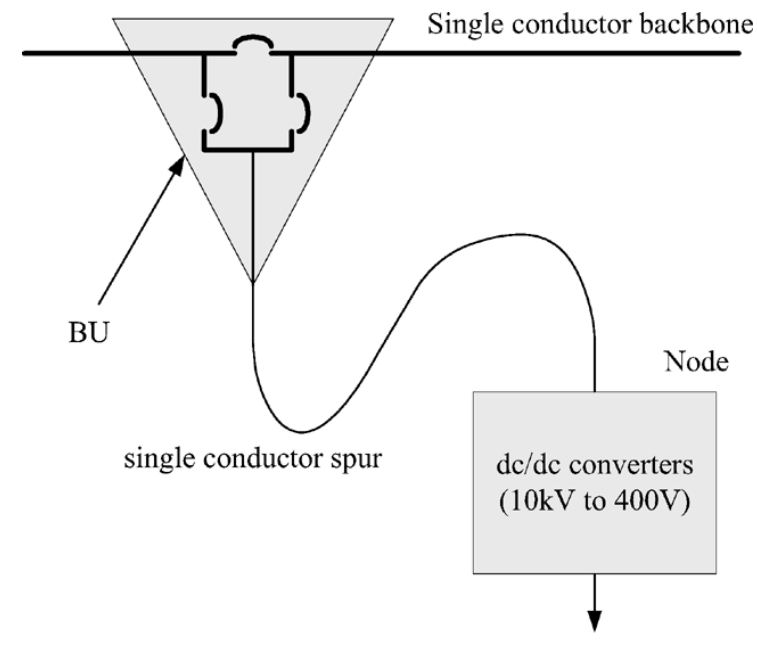

To science instruments

Fig. 2. BU to science node connection.

by the science node loads. It is at the science node that science users, the end users, are served power.

The BU has a series power supply with the simplest feasible implementation for the controls. It has been determined that it is possible to design a system that has only a $50 \%$ chance of requiring a service visit to a BU for maintenance or repair once in the 30-year life of NEPTUNE. However, the simplicity of design that is necessary to achieve the required level of reliability results in a design where there is no communication system access to the BU. Consequently, the voltages, currents and even the status of the switches within a BU are not directly known to the NEPTUNE monitoring system on shore. In order to compensate for the lack of power system data from the BUs, a state estimation algorithm has been developed to determine the voltages at the BUs [7]. The state estimation algorithm allows for the estimation of the BU voltages based on the voltage and current measurements at the science nodes, as well as the shore stations in conjunction with the information from the assumed topology of the system.

\section{State Estimation for the NePtune System}

In a conventional terrestrial power system, data is collected via the Supervisory Control And Data Acquisition (SCADA) system and state estimation is performed by the Energy Management System (EMS). The NEPTUNE equivalent of a SCADA and EMS system is called the Power Monitoring And Control System (PMACS). It is within the PMACS software that the state estimation functions are performed.

The basis of state estimation is to relate the values measured within the system to the unknown state variables, as shown in

where

$$
Z^{\text {meas }}=H^{*} x+\varepsilon
$$

$Z^{\text {meas }}$ column vector of measured values;

$H$ matrix of coefficients relating the known and unknown variables, based on system topology;

$x \quad$ estimated BU voltages;

$\varepsilon \quad$ measurement errors. 


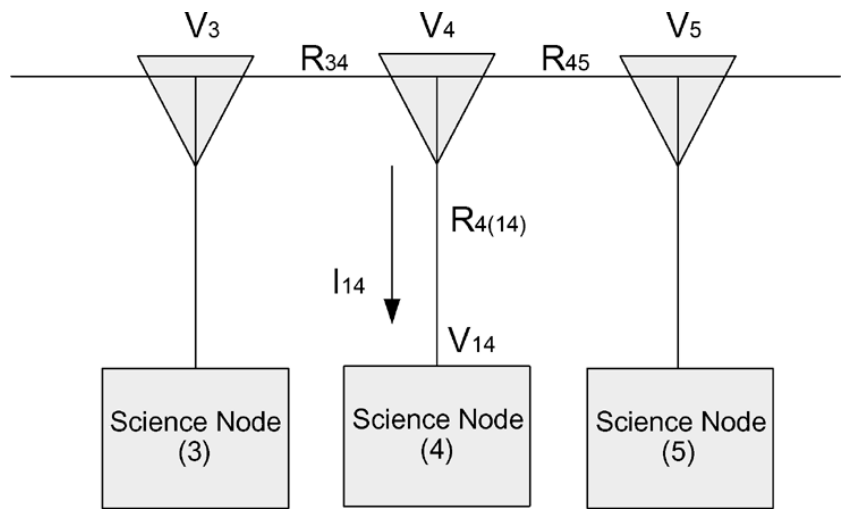

Fig. 3. Three BUs and three science nodes.

Through the use of Kirchhoff's current and voltage laws, it is shown that the current flowing into a science node and the science node voltage can be expressed in terms of the assumed topology of the system and the unknown BU voltages. Fig. 3 will be used as an example of how the measurements made at a science node are expressed in terms of the unknown values, BU voltages, and line resistances.

Using (2) and (3) as a framework, the voltage and current measurements at each of the science nodes can be expressed as

$$
\begin{aligned}
I_{14} & =\left(\frac{V_{4}-V_{5}}{R_{45}}-\frac{V_{3}-V_{4}}{R_{34}}\right) \\
V_{14} & =V_{4}-\left(\frac{V_{4}-V_{5}}{R_{45}}-\frac{V_{3}-V_{4}}{R_{34}}\right) * R_{4(14)}
\end{aligned}
$$

where

$V_{3}-V_{5}$ unknown BU voltages;

$V_{14} \quad$ measured science node voltage;

$I_{14} \quad$ measured science node current;

$R_{34}, R_{35}$ backbone resistances;

$R_{4(14)} \quad$ spur resistance.

While it is possible to express any single BU voltage as a function of only the associated science node, this method does not take advantage of the redundant measurements in the system. In order to account for under constrained systems where there are more measured values than unknown values, it is helpful to construct an expression that gives the maximum likelihood of the unknown values through a weighted least squares (WLS) calculation (4)

$$
J(x)=\left(Z^{\text {meas }}-f(x)\right)^{T}\left(R^{-1}\right)\left(Z^{\text {meas }}-f(x)\right)
$$

where

$f(x) \quad H * x$ calculation of the measured values based on the $\mathrm{BU}$ voltages

$R^{-1}$ inverse of diagonal matrix of measurement variances

By expanding (4) and calculating $\nabla J(x)=0$, the WLS algorithm is given by (5)

$$
x^{e s t}=\left(H^{T} R^{-1} H\right)^{-1}\left(H^{T} R^{-1} Z^{\text {meas }}\right) .
$$

One advantage of the formulation of (5) is that if any single measurement is lost it is still possible to determine the voltages at every BU. Furthermore errors that arise in the measurements due to measurement error are filtered in the WLS calculation. Another advantage of the WLS algorithm for NEPTUNE is that due to the linearity of (2) and (3) an iterative calculation is not required. While it is possible to obtain multiple or no solutions for a set of linear equations, this has not been an issue for NEPTUNE.

The general formulation of (2) and (3) is valid for all sections of NEPTUNE since BUs only exist where there is a science node. The equations may change slightly based on the number of adjacent BUs, i.e., number of terms, but the general form of (2) and (3) remains consistent.

Equation (5) gives the WLS approximation of the voltages at the BU's. If the assumed topology of the system is correct and there is no measurement error, then (5) will yield the exact voltages at the BUs. When measurements containing a Gaussian error with a nonzero variance are used in conjunction with (5) to estimate the BU voltages, the values will not be exact but are more reliable than a single measurement due to the filtering of the least squares fit.

In a system where the topology is known it is possible to approximate the variance of the measurement error using the estimated BU voltages. In order to approximate the variance of the measurement error present in the system, a comparison is made between the measured values and the measured values as calculated from the estimated BU voltages, $f(x)$, the difference is indicated by the mean absolute residual (6)

$$
\begin{aligned}
R_{\text {mar }} & =\left(\frac{1}{n}\right) * \sum_{i=1}^{n}\left|z_{i}-H_{i} x^{e s t}\right| \\
& =\left(\frac{1}{n}\right) * \sum_{i=1}^{n}\left|T_{i} * Z^{\text {meas }}\right|
\end{aligned}
$$

where

$H_{i} \quad i$ th row of matrix $H$;

$n \quad$ number of measured quantities;

T $\quad I-H\left(H^{T} R^{-1} H\right)^{-1} H^{T} R^{-1}$;

$T_{i} \quad i$ th row of $T$;

The mean absolute residual, $R_{\text {mar }}$, is the sum of a column vector with each of the elements containing the absolute value of the difference between the measured science node values and those calculated from the estimated branching unit voltages. When there is no measurement error, zero variance, in the system and the assumed topology is correct, the mean absolute residual will be zero. As the variance of the measurement error is increased from zero, the value of $R_{\mathrm{mar}}$ will begin to increase and the estimated $\mathrm{BU}$ voltages begin to deviate from the actual values.

If a breaker inside a BU is in a position other than expected, the voltages at the branching units will be estimated incorrectly. With the simultaneous presence of measurement and topological errors, the system operator and PMACS will be supplied with erroneous information. Without the correct information it is possible for the control actions by PMACS to result in undesired system conditions. For this reason, the topology processor component of PMACS uses the mean absolute residual as a basis for determining the current system topology. 


\section{BASIS OF TOPOLOGY IDENTIFICATION}

For the purposes of this study, there are two distinct classifications of system topology, i.e., the design topology and the operational topology. The design topology is the topology of the system as it is constructed, with all breakers closed and all lines in service. The operational topology is determined by the current status of breakers and combination of lines currently in service. If all breakers are closed with all lines in service, the operational topology and the design topology are identical. This section deals with the situation where the operational topology of the system is other than expected due to a breaker status error.

In terrestrial power systems there are redundant sources of information to determine the position of breakers, e.g., breaker auxiliary contacts, current measurements through the breaker, voltage measurements across a breaker, power flow along a line, and visual inspection results. In the absence of direct indications such as auxiliary contacts, conventional methods make use of indirect measurements, such as voltage differences and line flows, to perform the combined function of state estimation and topology identification [13]-[16]. The lack of comparable indications, direct or indirect, in the NEPTUNE systems requires a new method of topology error identification.

In order to accurately perform the state estimation and topology error identification, it cannot be assumed that the most significant contribution to the mean absolute residual originates with the topology error. There are three sources of error that contribute directly to the mean absolute residual: error from voltage measurements, error from current measurements, and error from topology errors. When the error from one source, e.g., voltage measurement, is much greater than the error source of interest, i.e., topology error, the source of interest is "masked." If there is no error in the voltage and current measurements, then the topology that gives a zero mean absolute residual would be the true topology. Due to the multiple sources contributing to the mean absolute residual and the associated masking effects, the mean absolute residual at a single operating point will not be sufficient to determine the operational topology.

In order to examine the system at multiple operating points, the system will be perturbed by varying the shore station voltages incrementally within a prescribed band. At each of the increments, data will be collected from the science nodes and the mean absolute residual calculated. The manner in which the mean absolute residual varies with respect to the shore station voltage variations will be the primary indicator of the system topology. By using the variation of the mean absolute residual, it will be possible to avoid the problems caused by masking.

When the assumed operational topology is the correct topology, it will initially be assumed that the mean absolute residual will vary in a roughly linear manner in the normal operating region, 5000-15000 $\mathrm{V}_{\mathrm{DC}}$ at all points in the system. Assuming that the variation will be linear, the sensitivity of the mean absolute residual with respect to changes in voltage at a single shore station is calculated by (7)

$$
\begin{aligned}
\frac{\partial R_{\mathrm{mar}}}{\partial V_{S S}} & =\left(\frac{1}{n}\right) * \frac{\partial}{\partial V_{S S}}\left(\sum_{i=1}^{n}\left|T_{i} * Z^{\text {meas }}\right|\right) \\
& =\left(\frac{1}{n}\right) * \sum_{i=1}^{n}\left(\operatorname{sign}\left(T_{i} * Z^{\text {meas }}\right) * T_{i} * \frac{\partial Z^{\text {meas }}}{\partial V_{S S}}\right)
\end{aligned}
$$

where $\partial R_{\text {mar }} / \partial V_{S S}$ is the sensitivity of the mean absolute residual and $V_{S S}$ is the voltage at the selected shore station.

The last term of (7), $\partial Z^{\text {meas }} / \partial V_{S S}$, requires that the sensitivities of each of the science node and BU voltages with respect to the shore station voltages be calculated. In order to calculate the required sensitivities, the power flow equations must be differentiated. The result is given by (8)

$$
\begin{array}{r}
-Y_{i(S S)} * V_{i}=\left\{\left(Y_{i i} * V_{i}+\sum_{j=1}^{k+2} Y_{i j} * V_{j}\right) \frac{\partial V_{i}}{\partial V_{S S}}\right. \\
\left.+\left(\sum_{\substack{j=1 \\
j \neq i}}^{k} Y_{i j} * V_{j} * \frac{\partial V_{j}}{\partial V_{S S}}\right)\right\}
\end{array}
$$

where

$Y_{i i} \quad i i^{t h}$ element of the bus admittance matrix;

$V_{i} \quad$ voltage at the $i^{t h}$ bus;

$k \quad$ total number of science nodes and BUs in the system.

Equation (8) is calculated with $i$ ranging from 1 to the number of science nodes, $k$, while setting SS equal to the node number of the shore station that is being varied. When $i$ is varied from 1 to the number of science nodes, the voltage at each of the science

$$
\begin{gathered}
\partial=\left[\begin{array}{c}
\frac{\partial V_{1}}{\partial V_{S S}} \\
\vdots \\
\vdots \\
\frac{\partial V_{k}}{\partial V_{S S}}
\end{array}\right], \quad B=\left[\begin{array}{cccc}
-Y_{1(S S)} & 0 & \cdots & 0 \\
0 & -Y_{2(S S)} & \cdots & 0 \\
\vdots & \vdots & \ddots & \vdots \\
0 & 0 & \cdots & -Y_{k(S S)}
\end{array}\right] \quad V=\left[\begin{array}{c}
V_{1} \\
\vdots \\
\vdots \\
V_{k}
\end{array}\right] \\
A=\left[\begin{array}{cccc}
Y_{1(1)} V_{1}+\sum_{j=1}^{k+2} Y_{1(j)} V_{j} & Y_{1(2)} V_{1} & \cdots & Y_{1(k)} V_{1} \\
Y_{2(1)} V_{2} & Y_{2(2)} V_{2}+\sum_{j=1}^{k+2} Y_{2(j)} V_{j} & \cdots & Y_{2(k)} V_{2} \\
\vdots & \vdots & \ddots & \vdots \\
Y_{k(1)} V_{k} & Y_{k(2)} V_{k} & \cdots & Y_{k(k)} V_{k}+\sum_{j=1}^{k+2} Y_{k(j)} V_{j}
\end{array}\right]
\end{gathered}
$$




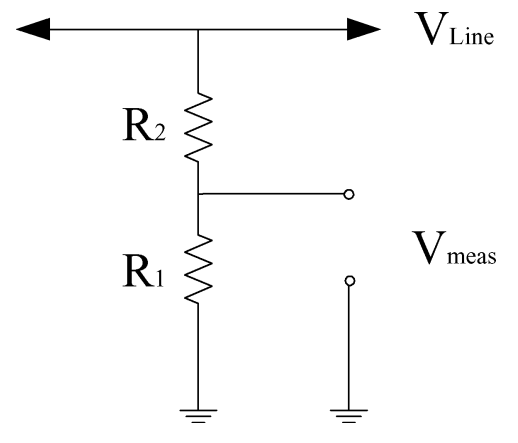

Fig. 4. Voltage measurement schematic.

nodes, as well as each of the BUs, with respect to the voltage at the shore stations can be calculated using (9). Equation (9) must be calculated at each operating point for each operational shore station. For the system in Fig. 1, there are maximally two shore stations in operation at any given time. We have that

$$
\partial=A^{-1} * B * V
$$

as defined in the equation shown at the bottom of the previous page.

Once the sensitivities of each of the science node and BU voltages are available, each of the elements of (7) can be calculated. Equation (7) gives the sensitivity of the mean absolute residual with respect to the shore station voltage for a system composed of only linear components. The next step is to determine if any of the power system devices used in NEPTUNE introduces nonlinearities into (7) and, if they do, determine their nonlinear characteristics. Three particular elements will be examined: the voltage measurement devices, current measurement devices, and DC-to-DC converters.

\section{A. Voltage Measurements Device Characteristics}

The power system devices that will be examined are those used to measure the voltage in the science nodes. These devices are connected as a voltage divider, as shown in Fig. 4.

For a voltage divider with ideal resistors, i.e., exact values, the measured voltage $V_{\text {meas }}$ is given by (10)

$$
V_{\text {meas }}=\frac{R_{1}}{R_{1}+R_{2}} V_{\text {Line }}
$$

Since it is not possible to obtain ideal resistors, it is necessary to introduce error into the values of resistance

$$
\begin{aligned}
& \widetilde{R}_{1}=R_{1}+\varepsilon_{1} R_{1}=R_{1}\left(1+\varepsilon_{1}\right) \\
& \widetilde{R}_{2}=R_{2}+\varepsilon_{2} R_{2}=R_{2}\left(1+\varepsilon_{2}\right)
\end{aligned}
$$

where

$\varepsilon_{1} \quad$ fractional error of resistor $\mathrm{R}_{1}$;

$\varepsilon_{2} \quad$ fractional error of resistor $\mathrm{R}_{2}$.

Substituting (11) into (10) yields (12)

$$
V_{\text {meas }}=\frac{\left(1+\varepsilon_{1}\right)}{\left(1+\varepsilon_{1}\right)+\frac{R_{2}}{R_{1}}\left(1+\varepsilon_{2}\right)} V_{\text {Line. }}
$$

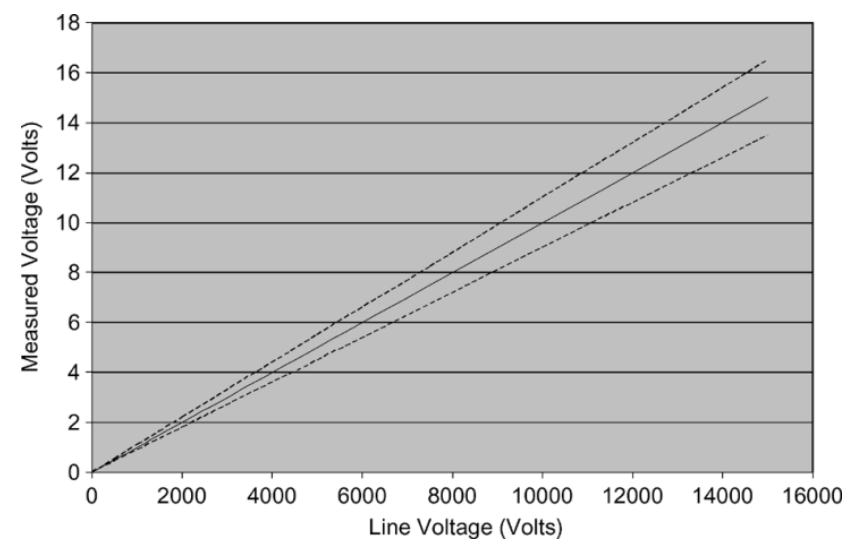

Fig. 5. Voltage error variation with line voltage.

In order to reduce the measured voltages $V_{\text {meas }}$ to usable control levels, the ratio of resistor values is set so that $R_{2} / R_{1}=$ 999. The result is a $\mathrm{V}_{\text {meas }}$ of between 5 and $15 \mathrm{~V}_{\mathrm{DC}}$ as $\mathrm{V}_{\text {Line }}$ varies from 5,000 to $15000 \mathrm{~V}_{\mathrm{DC}}$, i.e., the nominal operating range for the NEPTUNE power system.

For any sufficiently short interval of time, minutes to hours, the error of the resistor values can be considered constant. With the resistor error constant, the error in the measured voltage $\mathrm{V}_{\text {meas }}$ is reduced to a constant, multiplied by the line voltage $\mathrm{V}_{\text {Line, }}$, which yields a linear relationship between lines voltage and the measured voltage, shown in Fig. 5.

Fig. 5 shows a plot of the measurement error as the line voltage is increased from 0 to $15000 \mathrm{~V}$ for an isolated voltage measurement device. The solid line represents $V_{\text {meas }}$ when the values of $R_{1}$ and $R_{2}$ are exact. The two dashed lines represent $V_{\text {meas }}$ when $R_{1}$ and $R_{2}$ contain errors of $\pm 2 \%$. The key observation to be gained from examination of Fig. 5 is that as the line voltage is increased, the magnitude of the measured voltage error is also increased in a linear manner. A linear increase in the magnitude of line voltage will increase the magnitude of the mean absolute residual linearly. The result is that the voltage measurement devices do not introduce any nonlinear characteristics to (7).

\section{B. Current Measurement Device Characteristics}

The second set of power system devices to be examined includes those used to measure the current flowing into the science nodes. These devices are of a shunt design, as shown in Fig. 6.

Assuming that the resistive element, $\mathrm{R}$, will have an error, the following equations, (13) and (14), provide the calculated values of $\mathrm{V}_{\text {meas }}$ and the line current

$$
\begin{aligned}
V_{\text {meas }} & =I_{\text {Line(actual) }} * R(1+\varepsilon) \\
I_{\text {Line(calculated) }} & =(1+\varepsilon) * I_{\text {Line }(\text { actual })} .
\end{aligned}
$$

Equations (13) and (14) show that as the line current increases linearly, the magnitude of the measurement error and the corresponding mean absolute residual will also increase linearly. The result is that the current measurement devices do not introduce nonlinear characteristics to (7). 


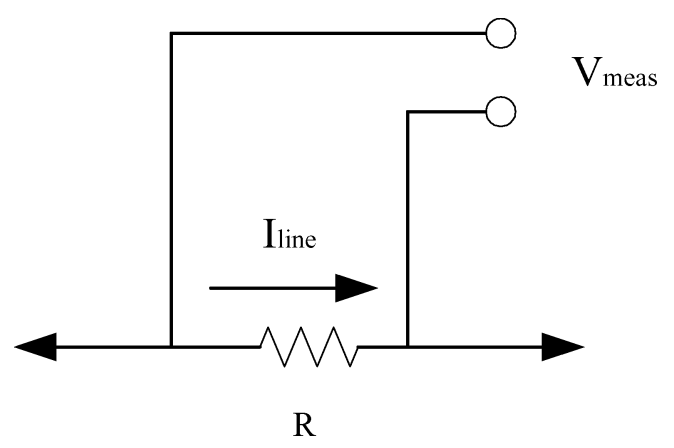

Fig. 6. Current measurement schematic.

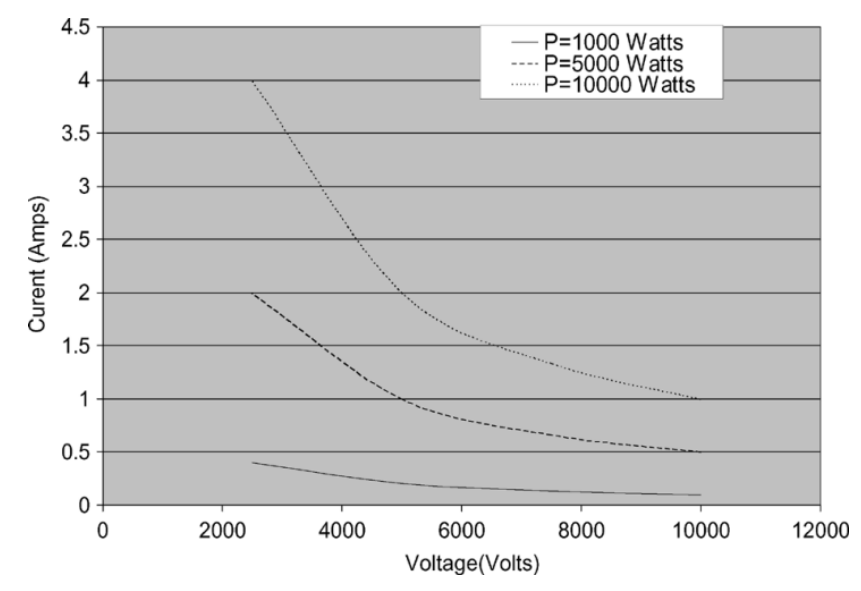

Fig. 7. Nonlinear converter characteristics.

\section{Converter Characteristics}

The third and final set of power system devices that will be examined is the DC-to-DC converters used at the science nodes. The converters step down the $10-\mathrm{kV}$ nominal backbone voltage to a constant $400 \mathrm{~V}$ for the end users. Since the output voltage is independent of the input voltage, the loads exhibit a constant power characteristic.

When the total system load is zero, the converters characteristics do not affect the system, resulting in a purely resistive and linear system. A zero system load, however, is not practical since the voltage and current measurements consume power in order to record and transmit their information to PMACS.

In order to examine the characteristics of a converter, the current versus voltage profile for a single isolated converter will be examined (see Fig. 7). As the load on the converter is increased from zero to the maximum value, nonlinear characteristics become more pronounced due to the hyperbolic relation between current and voltage for a constant power device. Fig. 7 shows the current versus voltage profile for three different power levels for a single isolated converter connected to an ideal voltage source.

Due to the slight nonlinear variations that arise from the converter dynamics, the ability of (7) to predict the variation of the mean absolute residual will depend on the loading of the various converters in the system. The result is that the converters have a nonlinear contribution to (7). The maximum nonlinear converter contribution is determined by the maximum system load. Thus, the maximum load scenario sets an upper bound for the converters' contribution to nonlinearity for a given operational topology.

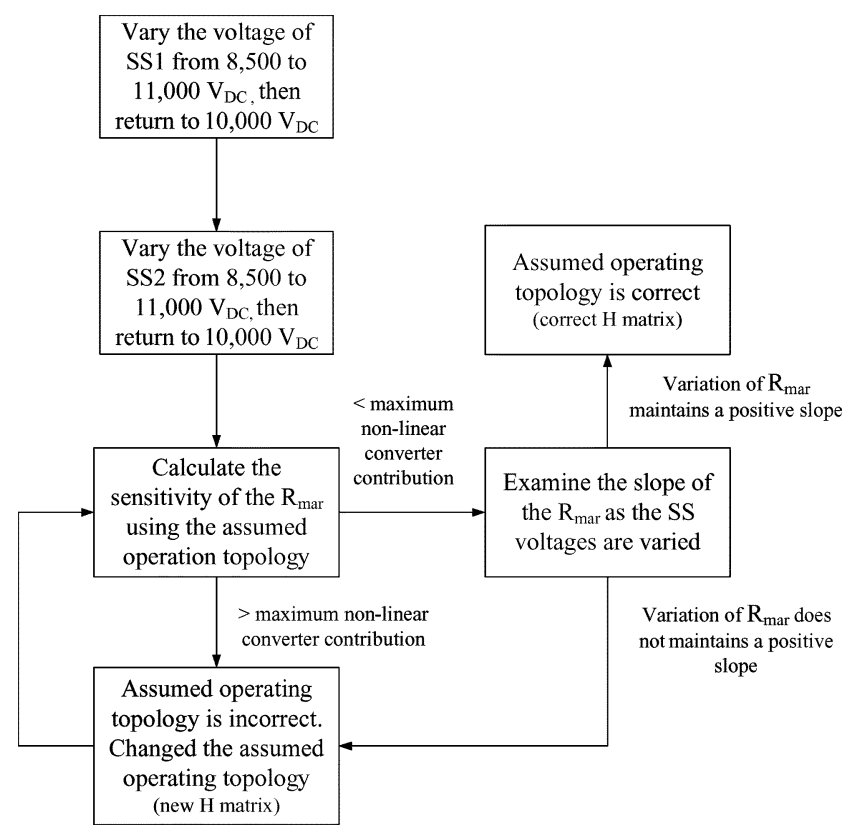

Fig. 8. Topology identification procedure.

\section{TOPOLOGY IDENTIFICATION METHOD}

With the correct topology assumed, the only nonlinear effects on the mean absolute residual are due to the converter dynamics. In order to take the converter dynamics into account, a maximum bound must be set on the nonlinear effects of the converter. The bounds correspond to the worst case nonlinear effect that converters can contribute to the mean absolute residual for a given operational topology.

Once the deviation from linearity is determined for the maximum system load, all load combinations of a lower level will cause less deviation. For the system of Fig. 1 the maximum variation from a linear approximation is $.847 \%$. Therefore, the assumed topology, for which the mean absolute residual varies by less than $.847 \%$ from linearity as shore station voltages are varied, indicates the correct system topology. All topologies that yield a variation higher than $.847 \%$ correspond to an incorrectly assumed operational topologies. Thus, the assumption of Section IV is confirmed.

The flow chart in Fig. 8 shows the procedure for determining the correct current operational topology of the system.

The complete procedure to detect the existence of a topology error and thereby determine the current operational topology is to first vary the voltage at shore station 1 from $8500 \mathrm{~V}_{\mathrm{DC}}$ to $11000 \mathrm{~V}_{\mathrm{DC}}$ while calculating the mean absolute residual at 500 $\mathrm{V}_{\mathrm{DC}}$ intervals. The voltage at shore station 1 is then returned to nominal and the same procedure is repeated with the voltage at shore station 2 . The mean absolute residuals are determined with the assumed topology. The following criteria applied.

1) The maximum variation of the mean absolute residual must be less than the value that is bounded by the characteristics of the converters at maximum system load.

2) The variation of the mean absolute residual must maintain a positive slope throughout the voltage range.

If either of the above two criteria is not met, a topology error is indicated. In order to determine the current operational 
topology, the mean absolute residuals are recalculated with different assumed operational topologies, $H$ matrices, until the $H$ matrix that satisfies the two criteria is found. The $H$ matrix that satisfies the two requirements represents the correct operational topology. When the mean absolute residuals are recalculated for each possible operational topology, it is not necessary to vary the shore station voltages again; the same measurements data can be used that was collected for the initial test.

As discussed earlier, masking is a condition that can result in an incorrect operational topology. By examining the mean absolute residual at multiple operating points, the proposed method ensures that masking does not interfere with topology identification. When the mean absolute residual is examined at a single operating point based on the magnitude criteria, masking occurs when multiple sources contribute to the magnitude. The proposed method examines a characteristic, i.e., sensitivity of the mean absolute residual, which only has two sources of contribution: topology errors and converter dynamics. The maximum contribution of the converter dynamics is calculated for a given operational topology and this value determines the upper bounds for the converter contribution. With the contribution from the only other source bounded, sensitivity effects from topology errors can be identified.

The masking of the mean absolute residual at a single operating point will be evident in cases where the second criterion is not met. When the variation of the mean absolute residual has a negative slope, it is an indication that measurement errors and topology errors are masking each other. While masking is a problem for some methods of topology identification, the proposed method uses the presence of masking to identify topology errors.

In order for the proposed method of topology identification to be applicable to any potential design topology, it is necessary to show that changes in the design topology do not introduce nonlinearities into the sensitivity of the mean absolute residual. To this end, (7) will be examined with respect to changes in the design topology. This is accomplished by operating only in the normal voltage range and at zero system load. Operation in the normal voltage range ensures the $\operatorname{sign}\left(T_{i} * Z^{\text {meas }}\right)$ term of (7) can be simplified as the term is a column vector of ones. Operation with zero system load allows for the nonlinear characteristics of the converters to be ignored. With the effects of the converters ignored, the system becomes a connection of purely resistive elements and the $\partial Z^{\text {meas }} / \partial V_{S S}$ term of (7) is a constant, as shown in (9). Under these conditions, (7) is a summation of constants, resulting in a constant slope. For various design topologies, the elements of the associated $Y$ matrix will affect the $\partial Z^{\text {meas }} / \partial V_{S S}$ term of (7). As a result, a summation of different constants for different design topologies yields different slopes. The result is that changes in the design topology do not introduce nonlinearities into the sensitivity of the mean absolute residual, and, as such, the method shown in Fig. 8 is valid for all potential design topologies.

\section{RESULTS OF TOPOLOGY IDENTIFICATION}

In order to test the method of topology identification presented in Section V, two cases will be examined, both of which

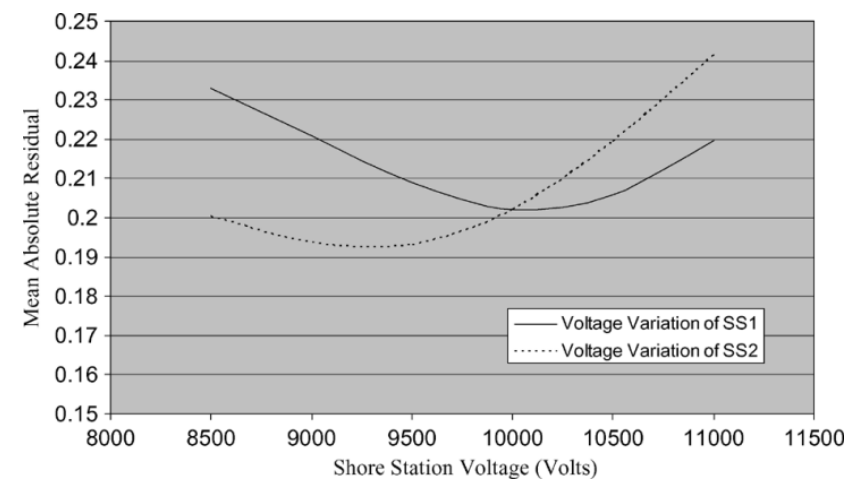

Fig. 9. Single topology error and incorrectly assumed operational topology.

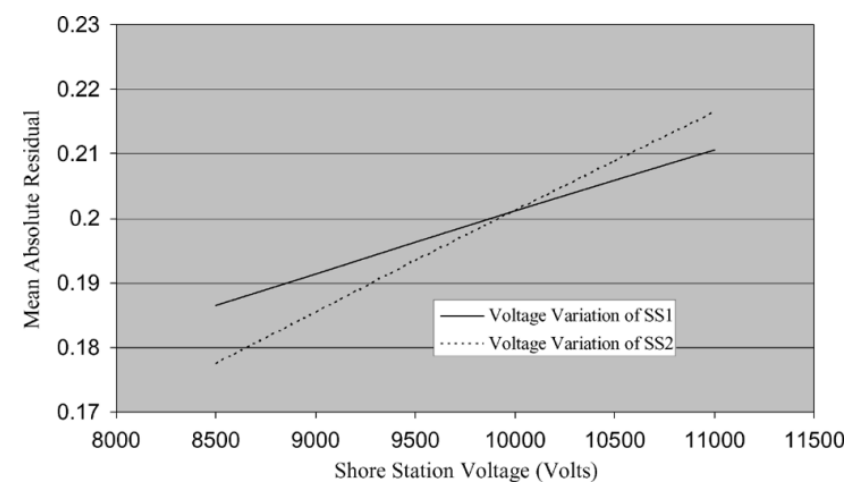

Fig. 10. Mean absolute residual variation with the correct topology.

are based on the design topology of Fig. 1. In the first case the method of Fig. 8 will be applied to a single topology error. In the second case, the proposed method will be applied to double topology errors.

In both cases there will be a $1 \%$ Gaussian measurement error as well as a single measurement with a gross measurement error of $30 \%$. These cases serve to examine the ability of the method to identify single and double topology errors in the presence of Gaussian as well as gross measurement errors.

\section{A. Case 1: Single Topology Error}

Fig. 9 shows the variation of the mean absolute residual, as calculated by (7), when there is a single topology error. Following the flow chart of Fig. 8, it is clear that the assumed operational topology is not correct since the variation of the mean absolute residual does not satisfy either of the two criteria detailed in Section V. Furthermore, the negative slope of the mean absolute residual indicates that there is masking of the mean absolute residual at certain individual operating points. As discussed in Section $\mathrm{V}$, the masking of individual operating points manifests itself as a negative slope and indicates the presence of a topology error.

When the $\mathrm{H}$ matrix associated with the correct operational topology is used in calculating (7), the mean absolute residual varies as shown in Fig. 10. The error between the variation shown in Fig. 10 and a linear variation as calculated by (7) is approximately $.697 \%$ over the given voltage range, less than the maximum converter contribution of $.848 \%$. Additionally, the variation of the mean absolute residual maintains a positive slope over the entire voltage range. Following the flow chart of 


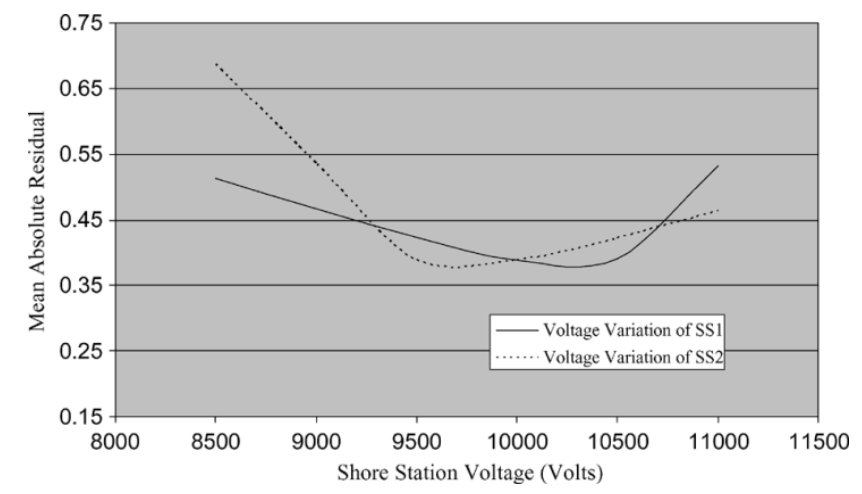

Fig. 11. Mean absolute residual variation with two topology errors.

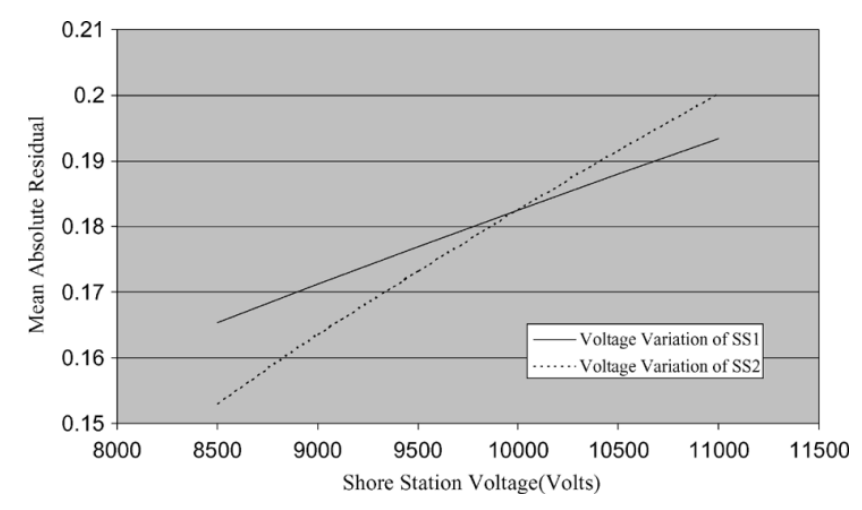

Fig. 12 . Mean absolute residual variation with the correct topology.

Fig. 8, these two conditions indicate a correctly assumed operational topology.

The inclusion of the single gross measurement error of $30 \%$ does not affect the linear characteristics of the mean absolute residual. The effect of gross measurement errors is to increase the magnitude of the mean absolute residual, in effect shifting the curve upward. The location of the gross measurement errors can be identified by examining the individual elements of the mean absolute residual. A gross measurement error presents itself as a value associated with an individual measurement that is much larger than the statistical average. Additionally, a Chi Squared test can be applied to the individual elements of the mean absolute residual to aid in the identification of gross measurement errors.

\section{B. Case 2: Double Topology Errors}

Fig. 11 shows the variation of the mean absolute residual, as calculated by (7), when there are double topology errors. Following the flow chart of Fig. 8, it is clear that the assumed operational topology is not correct since the variation of the mean absolute residual does not satisfy either of the two criteria detailed in Section V.

As with the case 1, the mean absolute residual is shown with the correctly assumed operational topology (see Fig. 12). Following the flow chart of Fig. 8, these two conditions indicate a correctly assumed operational topology.

In order to find the actual operational topology in the presence of topology errors, each of the possible topologies must be examined. This requires, at most, the calculation of (7) for each possible operational topology involving a single topology error.
For the system of Fig. 1, this requires calculating (7) 37 times for a single topology error, and $37^{2}$ times for double topology errors. While complete enumeration may be necessary, it is possible to reduce the search space by neglecting radial links. Radial links can be omitted from the search space since topology errors in these links have other gross indications, e.g., loss of science node information. For the system of Fig. 1, this reduces the number of calculations of (7) to 24 and $24^{2}$, for single and double topology errors, respectively. For triple topology errors and higher, the number of required calculations becomes prohibitive. These events will be extremely rare, however, for the NEPTUNE system.

\section{CONCLUSIONS}

This paper represents the continuation of work that has been in progress for over two years. The major contribution of this paper is a functional algorithm for topology identification in an interconnected direct current power system where the breaker status is not directly monitored. In addition to the lack of direct breaker indication, the system also lacks the indirect indications of breaker status used by many of the currently used topology identification methods.

Due to the high reliability design of the BUs, it will not be necessary to perform this topology identification method and perturb the system voltages very often. Determining how often it will be necessary to perturb the system and what, if any, adverse effects arise from perturbation of the system will be examined in a companion project, Monterey Accelerated Research System (MARS), prior to the deployment of the NEPTUNE system. Furthermore, the high reliability design of the BUs ensures that multiple topology errors will be rare.

The method of topology identification presented in this paper is not limited to under ocean applications. The same method could also be applied to future multiterminal/interconnected HVDC systems.

\section{ACKNOWLEDGMENT}

The authors would like to thank the reviewers for their insightful comments and highly meticulous work.

\section{REFERENCES}

[1] NEPTUNE Phase 1 Partners, (University of Washington, Woods Hole Oceanographic Institute, Jet Propulsion Laboratory, Pacific Marine Environmental Laboratory), "Real Time, Long-term Ocean and Earth Studies at the Scale of a Tectonic Plate: NEPTUNE Feasibility Study," Univ. Washington, Seattle, 2000.

[2] Proc. 3rd Int. Workshop on Scientific Uses of Submarine Cables and Related Technologies. Tokyo, Japan, Jun. 2003.

[3] J. Delaney, G. R. Heath, A. Chave, H. Kirkham, B. Howe, W. Wilcock, P. Beauchamp, and A. Maffei, "NEPTUNE Real-Time, Long-Term Ocean and Earth Studies at the Scale of a Tectonic Plate," in Proc. Oceans, 2001, MTS/IEEE Conf. and Exhib., vol. 3, pp. 1366-1373.

[4] B. Howe, H. Kirkham, and V. Vorperian, "Power system considerations for undersea observatories," IEEE Trans. Oceanic Eng., vol. 27, no. 2, pp. 267-274, Apr. 2002

[5] B. Howe, H. Kirkham, V. Vorperian, and P. Bowerman, "The Design of the NEPTUNE Power System," in Proc. Oceans, 2001, MTS/IEEE Conf. and Exhib., vol. 3, pp. 1374-1380.

[6] K. Schneider, C. C. Liu, T. McGinnis, B. Howe, and H. Kirkham, "Realtime control and protection of the NEPTUNE power system," in Proc Oceans, 2002, MTS/IEEE Conf. and Exhib., vol. 2, pp. 1799-1805. 
[7] K. Schneider, C. C. Liu, B. Howe, and H. Kirkham, "State estimation for the NEPTUNE power system," in Proc. IEEE PES Transmission and Distribution Conf., Dallas, TX, Sep. 2003.

[8] E. Handschin, F. C. Schweppe, J. Kohlas, and A. Fiechter, "Bad data analysis for power system state estimation," IEEE Trans. Power App. Syst., vol. PAS-89, no. 1, Jan. 1970.

[9] A. Monticelli, C. A. F. Murari, and F. F. Wu, "A hybrid state estimator: solving normal wquations by orthogonal transformations," IEEE Trans. Power App. Syst., vol. PAS-104, no. 12, Dec. 1985.

[10] M. B. D. C. Filho, A. M. L. da Silva, and D. M. Falcao, "Bibliography on power system state estimation (1968-1989)," IEEE Trans. Power Syst., vol. 5, no. 3, pp. 950-961, Aug. 1990.

[11] L. Mili, G. Steeno, F. Dobraca, and D. French, "A robust estimation method for topology error identification," IEEE Trans. Power Syst., vol. 14, no. 4, pp. 1469-1476, Nov. 1999.

[12] A. Abur and A. G. Expósito, Power System State Estimation Theory and Implementation. New York: Marcel Dekker, 2004.

[13] F. F. Wu and W. H. Liu, "Detection of topological errors by state estimation," in Proc. IEEE Winter Meeting, 1988, Paper 216-4.

[14] K. A. Clements and P. W. Davis, "Detection and identification of topology error in electric power system," IEEE Trans. Power Syst., vol. 3 , no. 4, pp. $176-183$, Nov. 1988 .

[15] I. S. Costa and J. A. Leao, "Identification of topology errors in power system state estimation," IEEE Trans. Power Syst., vol. 8, no. 4, pp. 1531-1538, Nov. 1993.

[16] A. Abur and M. K. Celik, "Topology error identification by least absolute value state estimation," in Proc. 7th Electrotechnical Conf., vol. 3, Apr. 1994, pp. 972-975.

[17] P. R. Bevington and D. K. Robinson, Data Reduction and Error Analysis for the Physical Sciences. New York: WCB/McGraw-Hill, 1992.

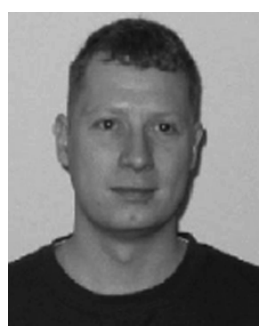

Kevin Schneider (S'03) received the B.S. degree in physics in 2001 and the M.S. degree in electrical engineering in 2002, both from the University of Washington, Seattle, where he is currently pursuing the Ph.D. degree in electrical engineering.

He received the Grainger Graduate Fellowship from the University of Washington in 2002 and 2003. His main area of research is the NEPTUNE power system.

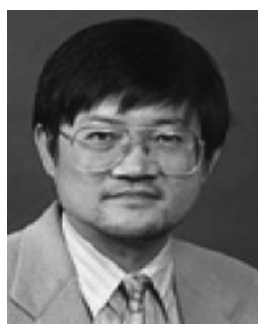

Chen-Ching Liu (F'94) received the B.S. and M.S. degrees in electrical engineering from The National Taiwan University, Taipei, Taiwan, R.O.C, and the $\mathrm{Ph} . \mathrm{D}$. degree from the University of California, Berkeley.

$\mathrm{He}$ is currently a Professor of Electrical Engineering and Associate Dean of Engineering at the University of Washington, Seattle. He also serves as Director of the Advanced Power Technologies (APT Center at the University of Washington).

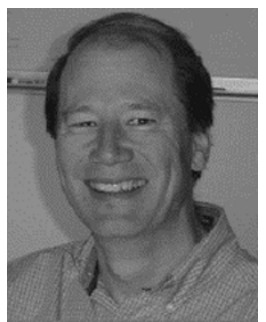

Bruce Howe (M'02) received the B.S. degree in mechanical engineering and the M.S. degree in engineering science in 1978 from Stanford University, Stanford, CA, and the Ph.D. degree in oceanography in 1986 from the Scripps Institution of Oceanography, University of California, San Diego.

While at Stanford University, he developed laser Doppler velocimetry (LDV) instrumentation for air-sea interaction experiments. From 1979 to 1981 , he was a Research Associate at the Institute Für Hydromechanik, Universität Karlsruhre, Karlsruhre, Germany, working on LDVs for use in the atmospheric boundary layer. While at Scripps Institution of Oceanography and since then, he has worked on ocean acoustic tomography, most recently on the Acoustic Thermometry of Ocean Climate (ATOC) project. He is presently a Principal Oceanographer at the Applied Physics Laboratory and a Research Associate Professor in the School of Oceanography, both at the University of Washington. His current interest is in cabled seafloor observatories, specifically the NEPTUNE project focused on the Juan de Fuca Plate. 